Universidad

:

BIBLIOTECA

Document downloaded from the institutional repository of the University of Alcala: https://ebuah.uah.es/dspace/

This is a postprint version of the following published document:

Zapata, F., Ortega-Ojeda, F.E. \& García-Ruiz, C., 2017. Revealing the location of semen, vaginal fluid and urine in stained evidence through near infrared chemical imaging. Talanta, 166, pp.292-299.

Available at https://doi.org/10.1016/i.talanta.2017.01.086

(C) 2017 Elsevier
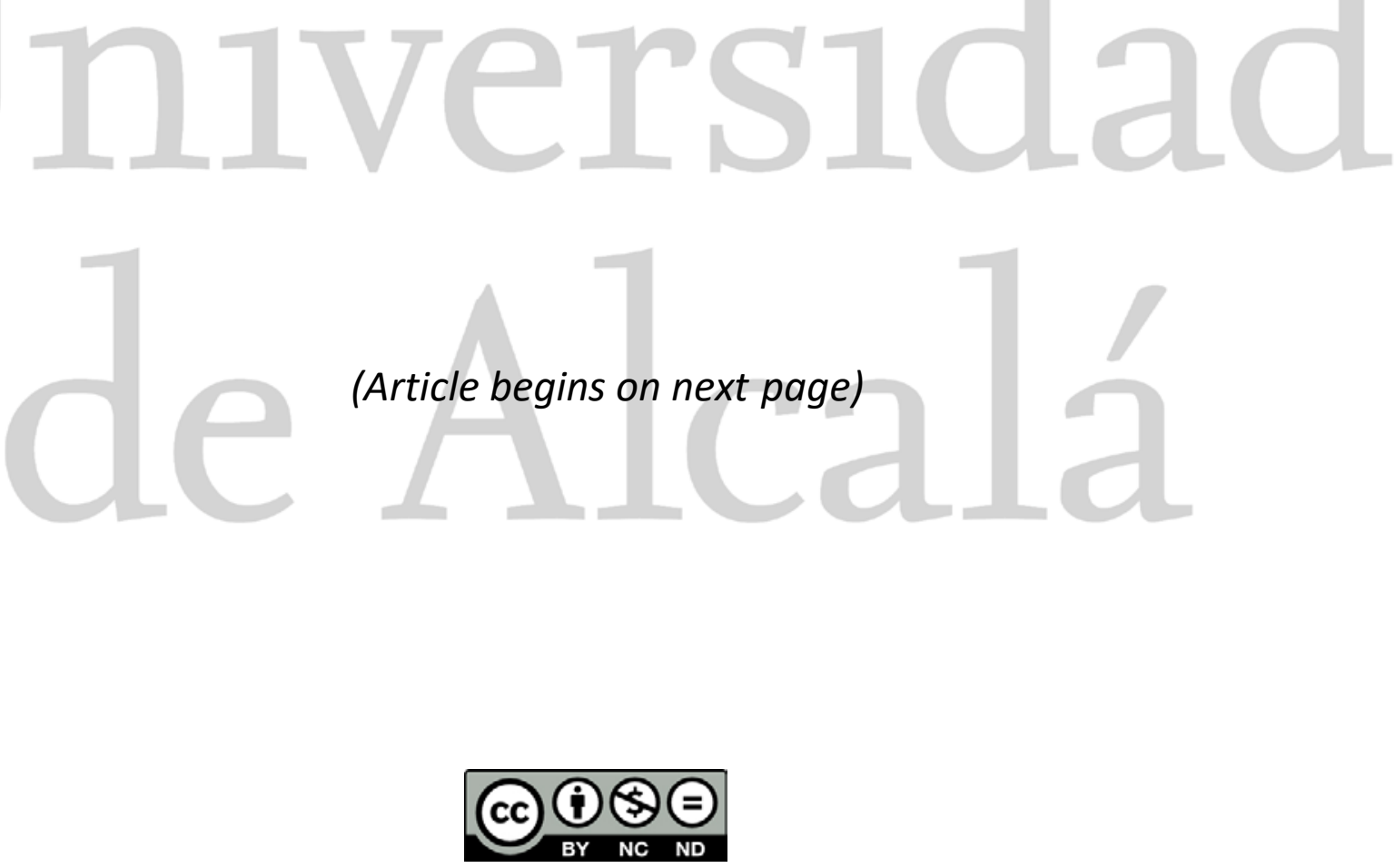

This work is licensed under a

Creative Commons Attribution-NonCommercial-NoDerivatives

4.0 International License. 


\title{
Revealing the location of semen, vaginal fluid and urine in stained evidence through near infrared chemical imaging
}

\author{
Félix Zapata, Fernando E. Ortega-Ojeda, Carmen García-Ruiz ${ }^{*}$
}

Department of Analytical Chemistry, Physical Chemistry and Chemical Engineering and Institute of Research in Police Sciences (IUICP), University of Alcalá, Ctra. Madrid-Barcelona km 33.600 28871 Alcalá de Henares (Madrid), Spain

Email: carmen.gruiz@uah.es; felix.zapata@uah.es

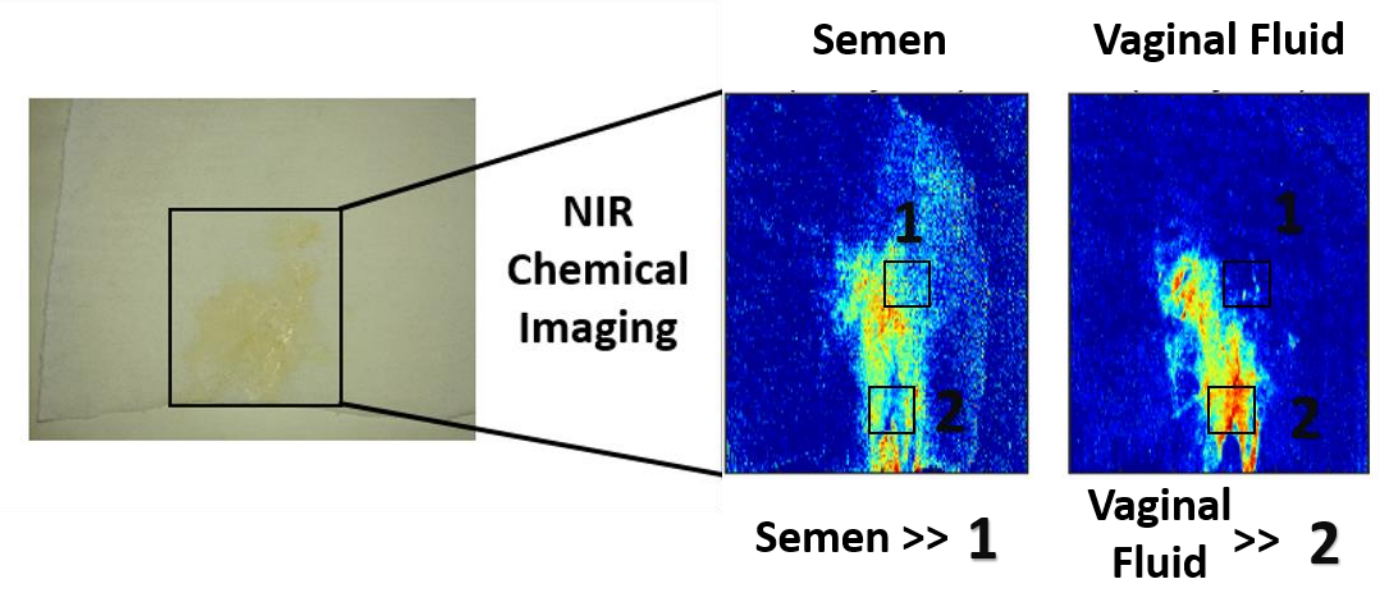

Cite: F. Zapata, F. E. Ortega-Ojeda, C. García-Ruiz, Revealing the location of semen, vaginal fluid and urine in stained evidence through near infrared chemical imaging, Talanta 166 (2017) 292-299. DOI: $\underline{\text { 10.1016/j.talanta.2017.01.086 }}$

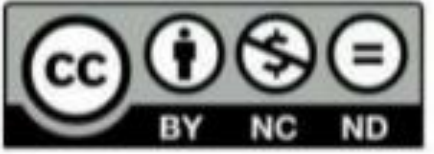




\begin{abstract}
Crime scene investigation (CSI) requires the ultimate available technology for a rapid, non-destructive, and accurate detection of a wide variety of evidence including invisible stains of bodily fluids. Particularly crucial is the discrimination of semen in stained evidence from sexual abuse cases. This is because those evidence have high odds of containing the DNA from the aggressor. To this aim, we demonstrated the potential of near infrared hyperspectral imaging (NIR-HSI) to make visible stains of semen, vaginal fluid, and urine on fabrics, which lays the bases to face the challenging visualization and discrimination of semen within bodily fluids mixtures. Combining the NIR-HSI data and simple chemometric techniques such as principal component analysis and classical least squares regression, we have revealed the location of semen, vaginal fluid and urine in bodily fluids stained evidence.
\end{abstract}

Keywords: ATR-FTIR; Semen; Urine; Vaginal fluid; Mixtures of bodily fluids; Superabsorbent pads. 


\section{Introduction}

The utilization of highly informative up-to-date analytical techniques for crime scene investigation in forensics seems evident to non-scientific people to such an extent that mostly think, influenced by TV series and brilliant super-detective characters, that forensic investigation is immediate and fool-proof. Nevertheless, despite being fiction, these series, showing 'in situ' fool-proof instrumentation suitable for instantly identifying any evidence, might be just futuristic. In fact, novel significant and highly useful approaches and methodologies towards that aim are being developed and implemented just from the first step in the crime investigation process, i.e., evidence recovery, as anticipated in the auspicious 'CSI The Hague' project [1].

Evidence recovery usually deals with the detection of minute, concealed and invisible traces, which require the expert and thorough inspection of forensic investigators. Different methodologies, devices and tests that assist the forensic inspection are currently being used for both location and identification purposes. Regarding bodily fluids evidence, UV light sources and chemical tests are well-known and well-established methodologies in the forensic community for the detection of stains of bodily fluids such as blood, semen, saliva or urine [2-4]. However, most of these tests are only presumptive, i.e., do not provide an unequivocal identification of the fluid. In addition, most of these tests are destructive, which is a huge disadvantage for bodily fluids, especially for those containing deoxyribonucleic acid (DNA), so useful for subsequent genetic analysis [2-4].

The novel technique called near infrared hyperspectral imaging (NIR-HSI) seems to be a promising approach to reach this evasive goal. This is due to the capability of NIR-HSI of photographing the trace while characterizing its spectral profiles providing chemical information [5]. NIR radiation provides specific chemical information about the photographed samples improving their discrimination based on their chemical composition. That is a great advantage over both traditional visible (Vis) and ultravioletvisible (UV-Vis) photography, in which the discrimination of bodily fluids deals with various false positive substances and limitations for coloured backgrounds. This occurs because UV-Vis HSI identification is only supported by the colour and the non-specific UV-fluorescence of bodily fluids [6-8]. Although it is true that NIR spectroscopy is less specific than MIR for characterization and almost useless for interpretation purposes $[9,10]$, it might be selective enough to characterize and discriminate different molecules 
according to their either evident or slight NIR spectral differences. Evident spectral differences may involve the presence or absence of the overtone from certain vibration while slight spectral differences might be small shifts in the wavelength value of a certain band and its band-shape. To this aim, multivariate pre-processing and chemometric methods are encouraged to enhance the discrimination from the NIR-HSI data [10-13].

Preliminary useful studies showing the advantages of using HSI for blood stains identification have been reported [14-17]. Nonetheless, other extraordinarily significant bodily fluids involved in sexual crimes such as semen, vaginal fluid and urine, usually mixed, remain unexplored by NIR-HSI. Interestingly, some Raman or MIR spectroscopy studies that focused on the spectral identification of these bodily fluids have proved the successful discrimination among them based on their different chemical composition [1823]. However, those techniques target at a single point and thus, they lack imaging. Therefore, they do not enable the visualization of stains, but only the identification of one point in the stain, which is almost completely useless in mixtures. In fact, the detection of semen still remains challenging when it is mixed with vaginal fluid. The higher proportion and, consequently, the higher signal from vaginal fluid in relation to semen hinders the identification of semen, and ultimately provides no satisfactory genetic results.

This work proposes, for the first time, a comprehensive study examining the potential of NIR-HSI to locate and discriminate semen from vaginal fluid even in mixtures.

\section{Experimental}

\subsection{Samples}

In this work, we studied stains of semen, vaginal fluid, and urine from ten anonymous donors ( 5 women and 5 men), as well as a mixture of semen-vaginal fluid. All stains were prepared following the procedure reported in previous literature [23]. Briefly, $0.6 \mathrm{~mL}$ of semen or urine were dropped on pieces of $11 \times 8 \mathrm{~cm}^{2}$ cut from T-shirts made of $100 \%$ cotton, whereas vaginal fluid was directly placed on the cotton pieces by each donor. In addition, a mixture containing semen and vaginal fluid was prepared with the aim of testing the suitability of NIR-HSI to discriminate those regions containing semen from those containing vaginal fluid along the same stain. This mixture-stain was prepared by dropping $0.6 \mathrm{~mL}$ of semen over the cotton piece just after having been stained with 
vaginal fluid. This way of preparation was selected in order to create similar stains to those found in real evidence, in which the fabric already stained with vaginal fluid might be subsequently stained with the aggressor's semen. The size of every stain ranged between 2 and $3 \mathrm{~cm}$ in diameter. All stains were left to air dry overnight before being analysed. Altogether, we studied five stains per bodily fluid (one stain per donor).

\subsection{Instrumentation for HSI analysis}

We used a hyperspectral camera (Specim, Oulu, Finland) kindly provided by INFAIMON (Barcelona, Spain) working from 1000 to $2500 \mathrm{~nm}$ wavelengths to analyse the stains. The camera had a Mercury Cadmium Telluride (MCT) cryogenically cooled detector. The inbuilt software of the HSI system enabled collecting the data, and then saving it in binary hdr-raw ENVI format (Exelis, Boulder, Colorado, USA). The hyperspectral data collected for each sample yielded a 3D hypercube (x, y, $\lambda$ ) with 243 different wavelengths.

\subsection{Data analysis}

The hyperspectral data analysis was performed in Matlab (MathWorks, MA, USA) using specific in-house algorithm sequences assisted by some freely-available HYPER-Tools functions $[11-13,24]$. We designed the algorithm sequences to perform data preprocessing and data classification analysis. Briefly, this process consisted of three main tasks:

- Masking/Selecting the region of interest. In order to ensure the HSI images contained the stain, the images comprised the whole fabric area. However, some areas of the fabric containing useless information were removed to save both processing and data analysis time. The area containing the stain was selectively masked/selected using principal component analysis (PCA). A PCA was performed to each 3D-hypercube image (considering its 243 wavelengths). This enabled a rapid visualization of the stain due to the spectral differences of the bodily fluid with respect to the fabric. Interestingly, it is remarkable that most stains were highlighted using the second or third principal component even though they had not been still preprocessed. (The first principal component was always correlated with the whole cotton sample). That is, the study of the first three principal components was enough to reveal the stains over cotton whilst explaining the characteristic spectral differences of the bodily fluids with regard to the cotton. As explanatory draft, Figure 1 displays the colour map scores obtained for one stain of each bodily fluid using the principal component that correlates with the 
stain. For instance, for these samples, the stains of either semen or urine were revealed using the third principal component while the stain of vaginal fluid was revealed using PC2. This way to visualize stains was certainly impressive since some stains were invisible to the naked eye (i.e., in visible wavelengths), or even invisible at each NIR wavelength on its own. This fact demonstrated the great assistance of PCA when dealing with hyperspectral images since it performed, in only a few seconds, the multivariate analysis of the 3D-hypercube, looking for the major differences along the 243 wavelengths at once.
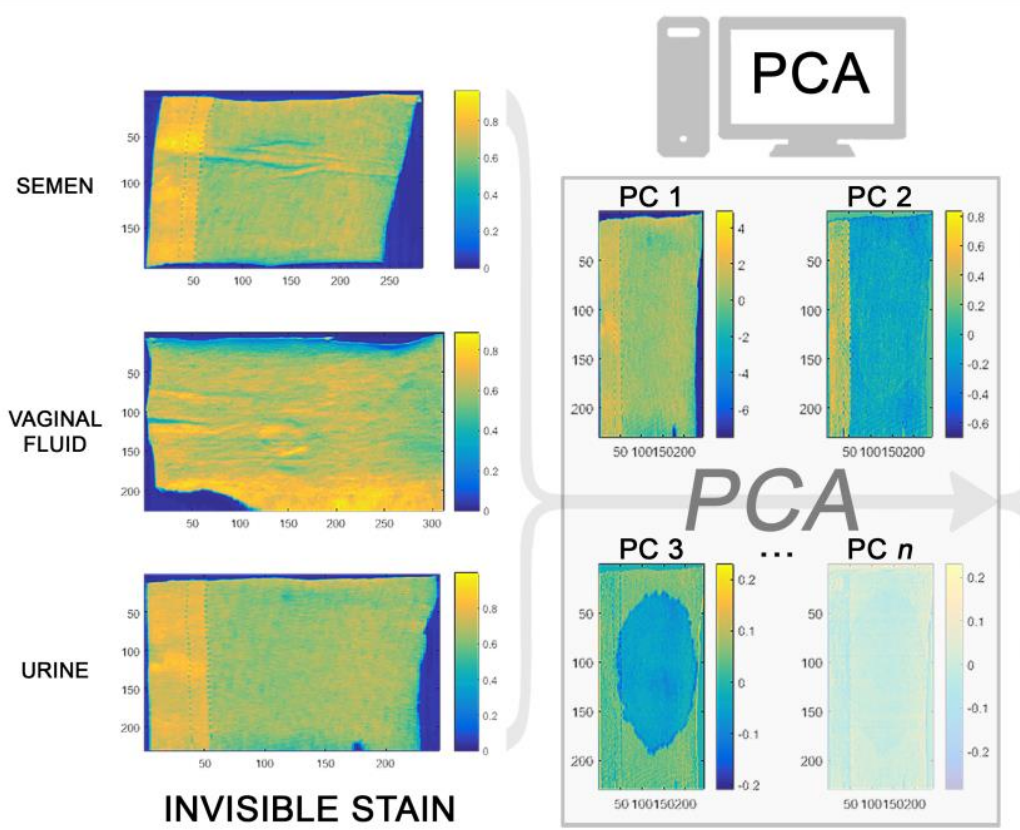

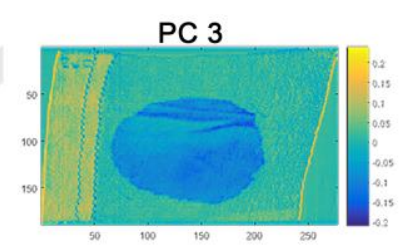

PC 2

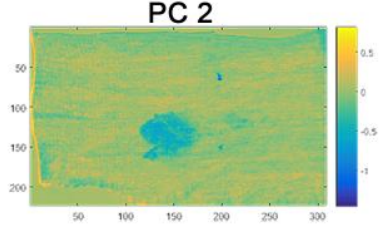

$\mathrm{PC} 3$

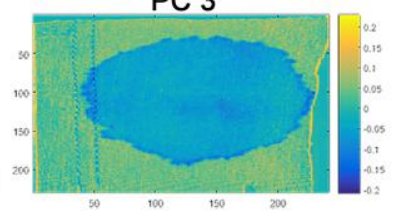

VISIBLE STAIN

Figure 1. The use of PCA for visualizing the stains of semen, vaginal fluid, and urine on cotton fabrics. One stain per bodily fluid is included. The stains of semen, vaginal fluid and urine are revealed through PC3, PC2 and PC3, respectively. The intermediate PCA colour maps for PC1, PC2 and PC3, shown as example, are those from the urine stain.

- Data Pre-processing prior to discrimination and classification analysis. After testing different alternatives, we selected the most appropriate pre-processing sequence as follows: Selection of the spectral range $(1270-2300 \mathrm{~nm})$, Standard Normal Variate (SNV) normalization, and Savitzky-Golay smoothing (2nd degree polynomial fitting using a symmetrical window of 5 points).

- Data classification to discriminate the bodily fluids and visualize the traces of semen even in mixtures. After proving the discrimination of stains from cotton and optimizing the pre-processing, the next step focused on verifying that the stains from each bodily fluid could be differentiated from each other according to their respective characteristic 
NIR spectrum. First, a PCA exploratory analysis containing the spectra collected from one of the stains of each bodily fluid was performed in order to check whether the stains of semen, vaginal fluid and urine can be discriminated through NIR spectroscopy. Finally, a classical least squares (CLS) classification analysis was carried out in order to improve the discrimination of these stains to such an extent that they may be discriminated and classified even in mixtures. To this aim, 50 representative spectra of both cotton and stains of bodily fluids (taken from 1 of the 5 stains per bodily fluid) were gathered into an ad-hoc home-made NIR spectral library. The selection of these representative spectra for each bodily fluid stain was facilitated by PCA (i.e. spectra were manually selected within the stain region delimited by PCA keeping in mind the need to cover the middle of the stain as well as the periphery in order to include the intra-variability of the bodily fluid along the stain). Afterwards, the other four stains of each bodily fluid were compared against the bodily fluids NIR spectral library as blind tests, as summarized in Table 1. Finally, a stain containing a mixture of semen-vaginal fluid was compared against the model in order to test the suitability of this methodology to classify mixture stains. This comparison was performed using a classical least squares regression (CLS) between the spectra from the pixels in each image and the NIR spectral library previously created. As a result, a matching value based on the CLS regression was provided for each pixel in the image to each class (cotton, semen, urine and vaginal fluid). By plotting this matching values, a coloured chemical map was visualized for each bodily fluid (besides cotton). The colour in every map ranged from blue (smallest value) to red (maximum value), indicating the estimated weight of bodily fluid in that pixel. In other words, a value close to 1 would imply a perfect matching between the spectrum from a red pixel and the NIR spectra from the library of that fluid. Interestingly, all samples tested displayed an intense red region when comparing with the correct bodily fluid spectral library, but a blue-to-yellow region when comparing with the incorrect bodily fluid. Furthermore, in order to numerically assess the classification power within a randomly selected region of a stain, the CLS results provided by that region to each class (cotton, semen, urine or vaginal fluid) are compared to each other. To this aim, the maximum weight value for the selected region at each coloured chemical map, is shown above every coloured map. In addition, the histogram containing the total number of pixels within the selected region, classified into the specific class with a certain value (i.e. every pixel with non-zero value to that class), is displayed below every coloured map. 
Table 1. Distribution of stains used to create the spectral library / to test the model.

\begin{tabular}{|l|l|}
\hline Stain & Used for \\
\hline Cotton blank & Library \\
\hline Semen stain donor 1 & Library \\
\hline Semen stain donor 2 & Blind test A \\
\hline Semen stain donor 3 & Blind test B \\
\hline Semen stain donor 4 & Blind test C \\
\hline Semen stain donor 5 & Blind test D \\
\hline Urine stain donor 1 & Library \\
\hline Urine stain donor 2 & Blind test A \\
\hline Urine stain donor 3 & Blind test B \\
\hline Urine stain donor 4 & Blind test C \\
\hline Urine stain donor 5 & Blind test D \\
\hline Vaginal fluid stain donor 1 1 Library \\
\hline Vaginal fluid stain donor 2 & Blind test A \\
\hline Vaginal fluid stain donor 3 & Blind test B \\
\hline Vaginal fluid stain donor 4 & Blind test C \\
\hline Vaginal fluid stain donor 5 & Blind test D \\
\hline Mixture (semen-vaginal fluid) stain & Blind test \\
\hline
\end{tabular}

\section{Results and discussion}

First of all, it was verified whether the stains from bodily fluids (semen, urine, and vaginal fluid) were active enough in NIR to be differentiated from each other according to their respective characteristic NIR spectrum. Although the NIR spectra from the three bodily fluids on cotton were quite similar (Figure 2), a thorough inspection revealed some slight differences at the spectral regions around 1500, 1600-1900, 1950 and 2100-2300 nm. 


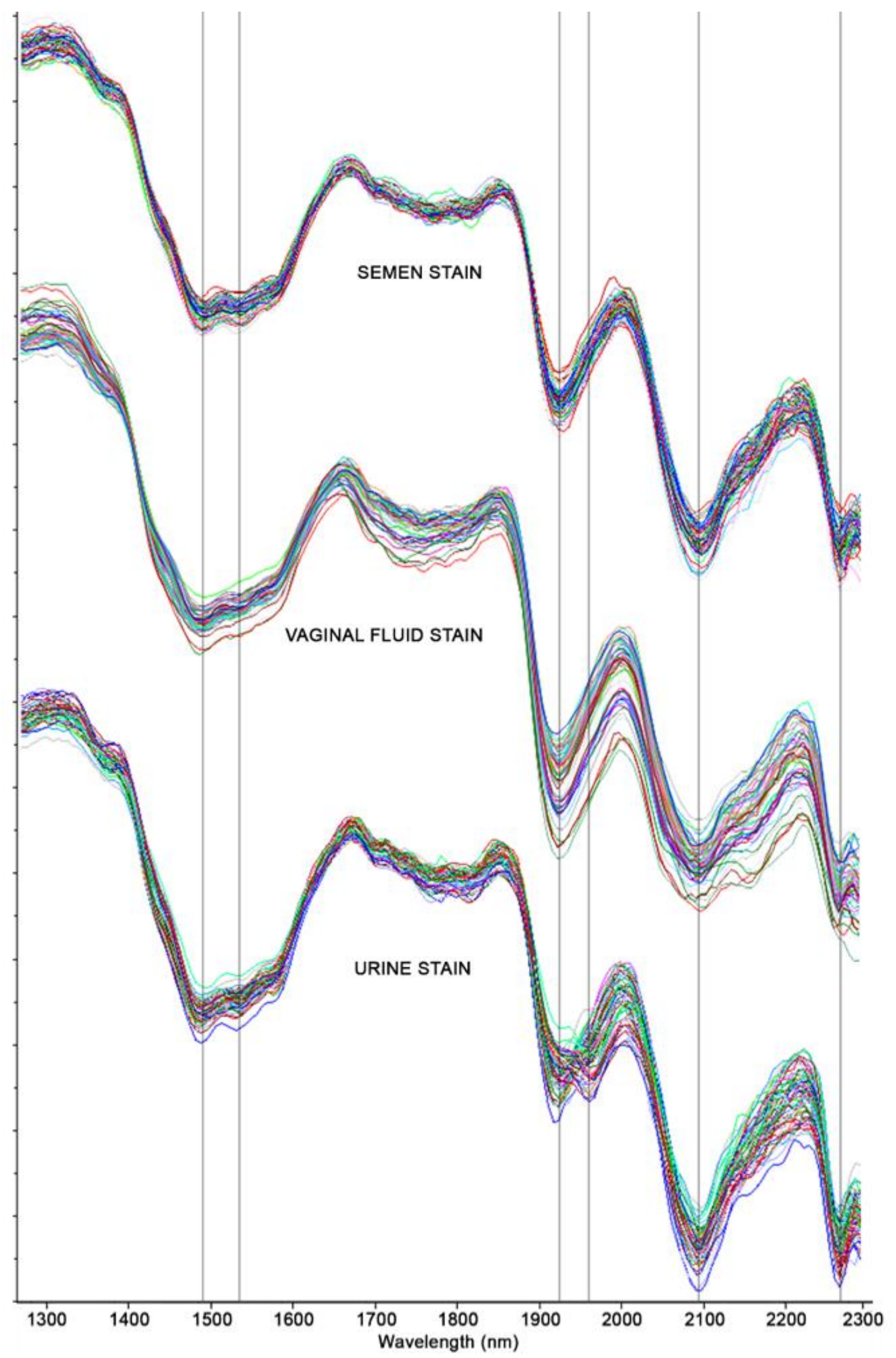

Figure 2. NIR spectra from stains of semen, vaginal fluid and urine on cotton fabrics from 1270 to $2300 \mathrm{~nm}$. 
Despite being negligible to the naked eye, a simple PCA study (see Figure 3) evidenced the significance of these slight differences to discriminate these stains. In fact, the scores corresponding to the spectra of bodily fluids, which are determined by the spectral loadings, were placed conforming slightly separated groups according to each bodily fluid. In brief, the spectra of vaginal fluid stains were distributed along negative values of PC1; the spectra of semen stains were distributed around zero and low positive values of $\mathrm{PC} 1$; and the spectra of urine stains were distributed along higher positive values of PC1. Consequently, the discrimination of stains from the three bodily fluids seems to be achievable using NIR spectroscopy supported by chemometrics.
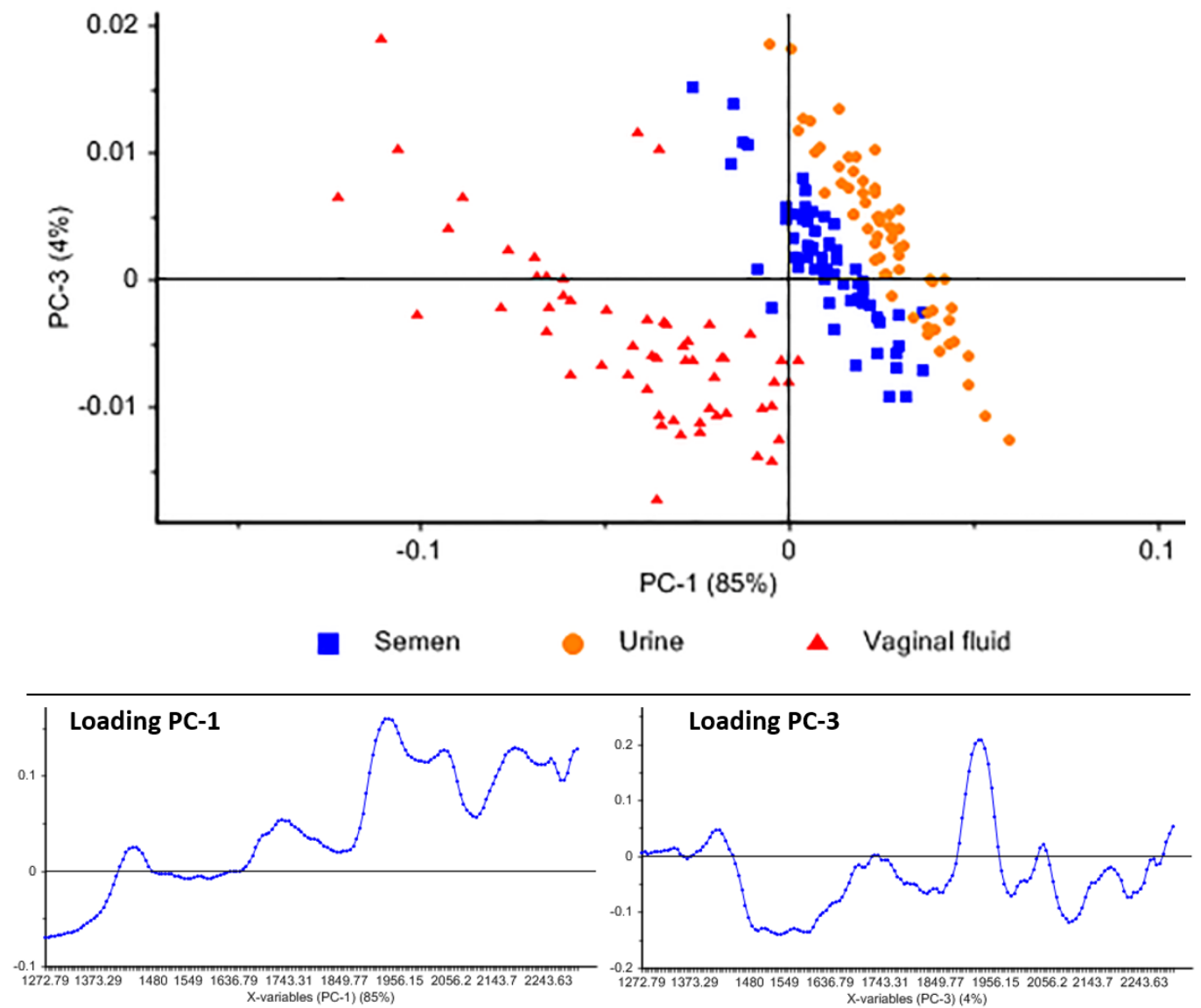

Figure 3. Two-dimension scores PCA plot along PC1 and PC3 considering the NIR spectra from stains of semen, vaginal fluid, and urine on cotton.

In order to improve the discrimination, a classification methodology based on CLS was performed by identifying the bodily fluid in a stain (blind sample) applying a particular level of certainty. As an example, Figure 4 summarizes the results for a semen stain used as blind sample. 


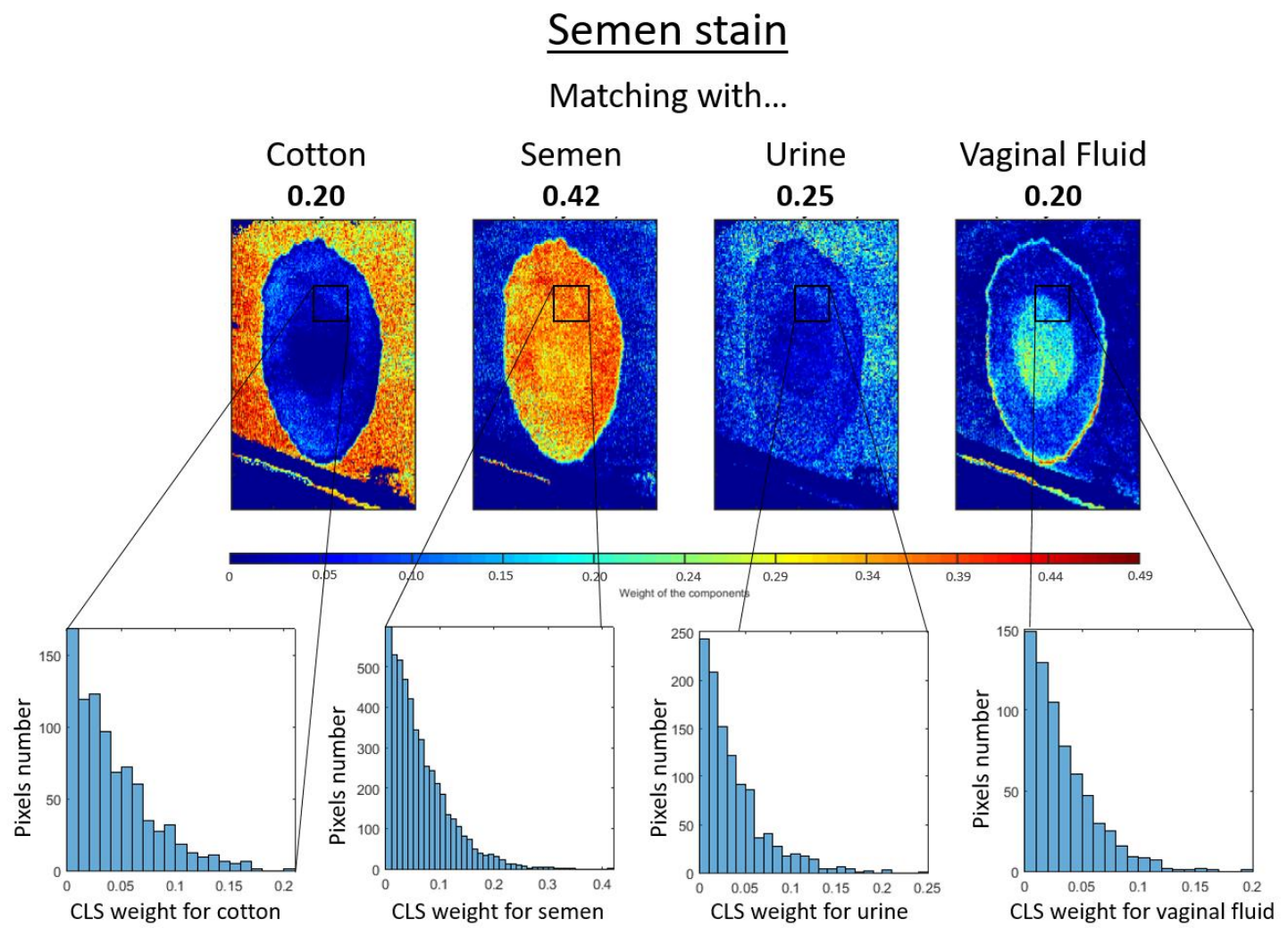

Figure 4. CLS classification model applied to a semen stain on cotton fabric. The CLS coloured maps for each class (cotton, semen, urine and vaginal fluid) are displayed. The maximum CLS weight values obtained for each class within the selected stained region is indicated above every colour map. The histogram containing the number of pixels within the selected region and their corresponding CLS weight to each class are provided below every colour map.

As expected, cotton, which is the background, was identified outside the stain as displayed in orange-to-red colours in the first subplot of Figure 4. Remarkably, cotton appears like surrounding the stain of bodily fluid, i.e., the bodily fluid provided characteristic spectral features which are distinctively different from those of cotton. In fact, regarding the selected region within the stain, the maximum weight value for cotton was 0.21 . In the same way, the histogram shows the small number of pixels within this region (870) classified as cotton with a low value from 0 to 0.21 . However, the most significant result involved the identification of the stain as semen as displayed in Figure 4, second subplot, by the orange-red area which perfectly defined the stain. In addition, the maximum weight semen value within the selected area (0.42) and the high number of pixels included in its histogram (4884) support the identification as semen. Negatively, the classification into vaginal fluid (Figure 4, fourth subplot) also provided the shape of the semen stain, which could lead to confusion. Fortunately, the low weight of vaginal fluid within the selected region ( 0.20 of maximum weight) and the low number of pixels 
(672) reduce its quantitative identification as vaginal fluid. In fact, semen and vaginal fluid were already reported to have similar composition [2], which would likely explain the noticeable similarities between their NIR spectra. Finally, urine (Figure 4, third subplot) was heterogeneously and vaguely accounted by the CLS model as shown in the dispersed pixels, mainly placed outside the stain. Nevertheless, a significant weight $(0.25)$ and number of pixels (1099), were provided for urine within the selected region, which also shows certain similarities between the NIR spectra of semen and urine. Accordingly, the identification of semen within a region must be supported by both the maximum classification weight value and the number of pixels. See Table 2 to check the maximum weight classification values as well as the number of pixels within the selected region for each class (cotton, semen, urine and vaginal fluid) for the other three stains of semen included in this study. Similar results were obtained for the four stains.

Table 2. CLS classification results for stains of semen, urine, vaginal fluid and the semen-vaginal fluid mixture. The first column of each class, called 'Max.', indicates the maximum intensity of the CLS classification model within the selected region to that class. The second column of each class called 'Pixels' specifies the total number of pixels from the selected region within the stain whose CLS classification intensity to that class was non-zero (i.e. those pixels that were classified into that class with a certain value).

\begin{tabular}{|c|c|c|c|c|c|c|c|c|}
\hline \multicolumn{9}{|c|}{ Number of pixels from the selected region $(20 \times 20)$ classified with a certain value as... } \\
\hline & \multicolumn{2}{|c|}{ Cotton } & \multicolumn{2}{|c|}{ Semen } & \multicolumn{2}{|c|}{ Urine } & \multicolumn{2}{|c|}{ Vaginal fluid } \\
\hline & Max. & $\begin{array}{c}\text { Pixels } \\
(\text { Int }>0) \\
\end{array}$ & Max. & \begin{tabular}{|c|}
$\begin{array}{c}\text { Pixels } \\
(\text { Int }>0)\end{array}$ \\
\end{tabular} & Max. & \begin{tabular}{|c|} 
Pixels \\
$($ Int $>0)$ \\
\end{tabular} & Max. & $\begin{array}{c}\text { Pixels } \\
(\operatorname{lnt}>0)\end{array}$ \\
\hline Semen stain A & 0.21 & 870 & 0.42 & 4884 & 0.25 & 1099 & 0.20 & 672 \\
\hline Semen stain B & 0.12 & 37 & 0.49 & 4683 & 0.17 & 977 & 0.17 & 370 \\
\hline Semen stain C & 0.25 & 1239 & 0.43 & 4272 & 0.31 & 1827 & 0.15 & 446 \\
\hline Semen stain D & 0.18 & 302 & 0.55 & 5405 & 0.20 & 1092 & 0.15 & 449 \\
\hline Urine stain A & 0.26 & 974 & 0.24 & 709 & 0.45 & 5018 & 0.12 & 153 \\
\hline Urine stain B & 0.40 & 1435 & 0.18 & 355 & 0.53 & 4081 & 0.10 & 72 \\
\hline Urine stain C & 0.16 & 196 & 0.13 & 382 & 0.42 & 5494 & 0.06 & 32 \\
\hline Urine stain D & 0.04 & 3 & 0.12 & 49 & 0.54 & 3998 & 0.14 & 242 \\
\hline Vaginal fluid stain A & 0.34 & 1232 & 0.19 & 1059 & 0.27 & 1429 & 0.62 & 2923 \\
\hline Vaginal fluid stain B & 0.30 & 693 & 0.17 & 606 & 0.21 & 679 & 0.41 & 4586 \\
\hline Vaginal fluid stain C & 0.28 & 1193 & 0.14 & 218 & 0.32 & 560 & 0.50 & 3703 \\
\hline Vaginal fluid stain D & 0.30 & 563 & 0.20 & 213 & 0.24 & 229 & 0.66 & 3661 \\
\hline Mixture (semen region) & 0.37 & 1866 & 0.41 & 2639 & 0.36 & 1797 & 0.22 & 707 \\
\hline Mixture (vaginal fluid region) & 0.09 & 31 & 0.36 & 1576 & 0.11 & 86 & 0.69 & 2122 \\
\hline
\end{tabular}

Similarly, we tested blind stains of urine and vaginal fluid using CLS analysis and similar images to those shown in Figure 4 were obtained (See Figures S1 and S2). Regarding the 
urine stain, besides cotton which appeared highly red-intense surrounding the stain, but with a low weight (0.26) and small number of pixels (974) within the selected region inside the stain, only urine (with a maximum weight value of 0.45 and 5018 pixels within the selected region) was properly quantified all over the stain. Neither the matching with semen (maximum weight value of 0.24 and 709 pixels within the selected region) nor with vaginal fluid (maximum weight value of 0.12 and 153 pixels within the selected region) provided quantitative results high enough for their identification.

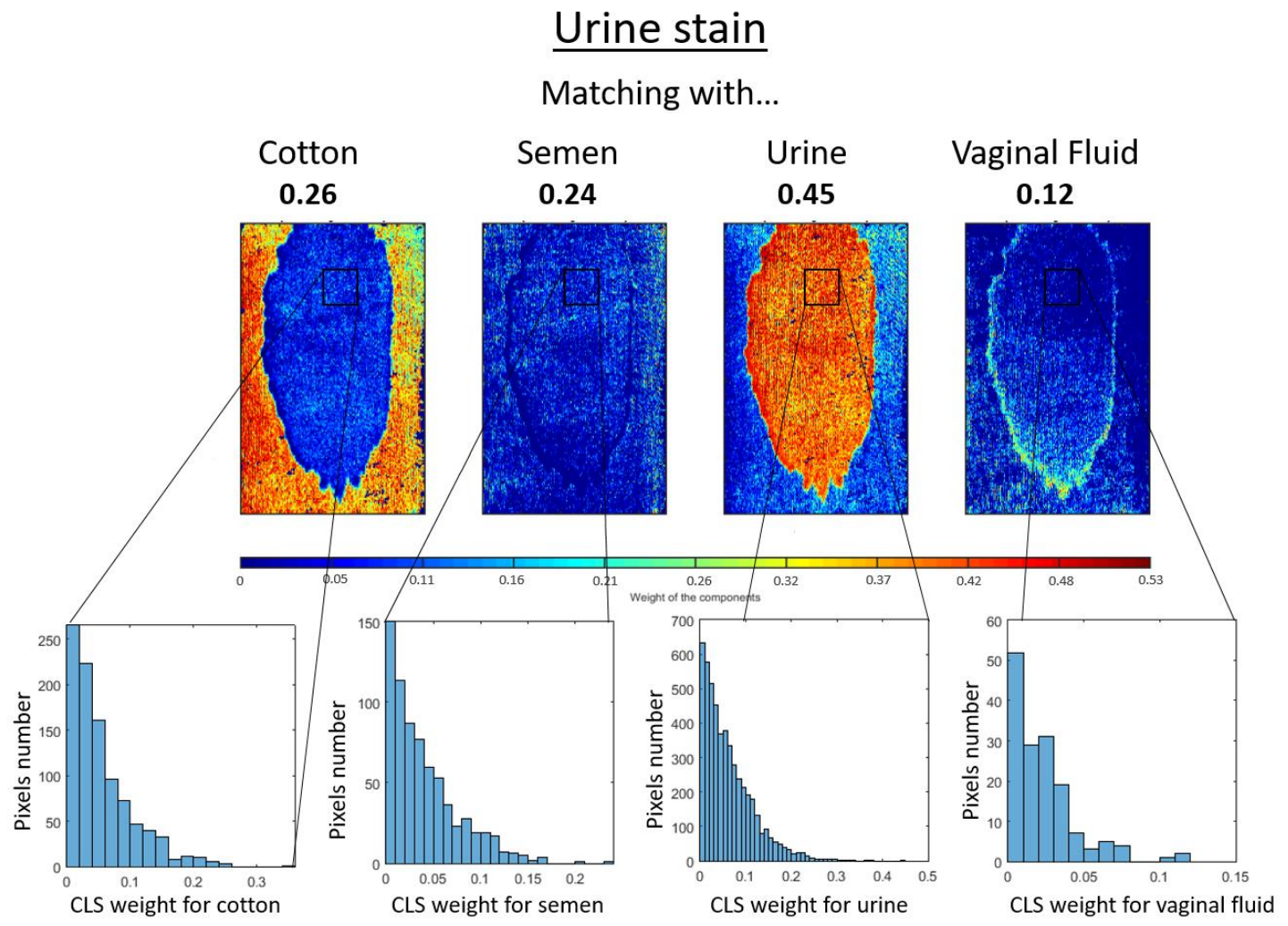

Supplementary Figure S1. CLS classification model applied to a urine stain on cotton fabric. The CLS coloured maps for each class (cotton, semen, urine and vaginal fluid) are displayed. The maximum CLS weight values obtained for each class within the selected stained region is indicated above every colour map. The histogram containing the number of pixels within the selected region and their corresponding CLS weight to each class are provided below every colour map.

With regards to the vaginal fluid stain (Figure S2), cotton was classified surrounding the stain, as expected, (and it provided a maximum weight value of 0.34 and 1232 pixels within the selected region) whereas vaginal fluid was classified throughout the stain, with 0.62 of maximum weight and 2923 pixels within the selected region. The matching with semen and urine within the selected region yielded maximum weight values of 0.19 and 0.27 , respectively; and 1059 and 1429 pixels, respectively. Although urine was randomly 
classified along the whole fabric, semen was mostly concentrated in a small well-defined region of the stain, as previously and contrarily occurred with the semen stain in comparison to vaginal fluid. Similar results were obtained for the rest of urine and vaginal fluid stains (see Table 2).

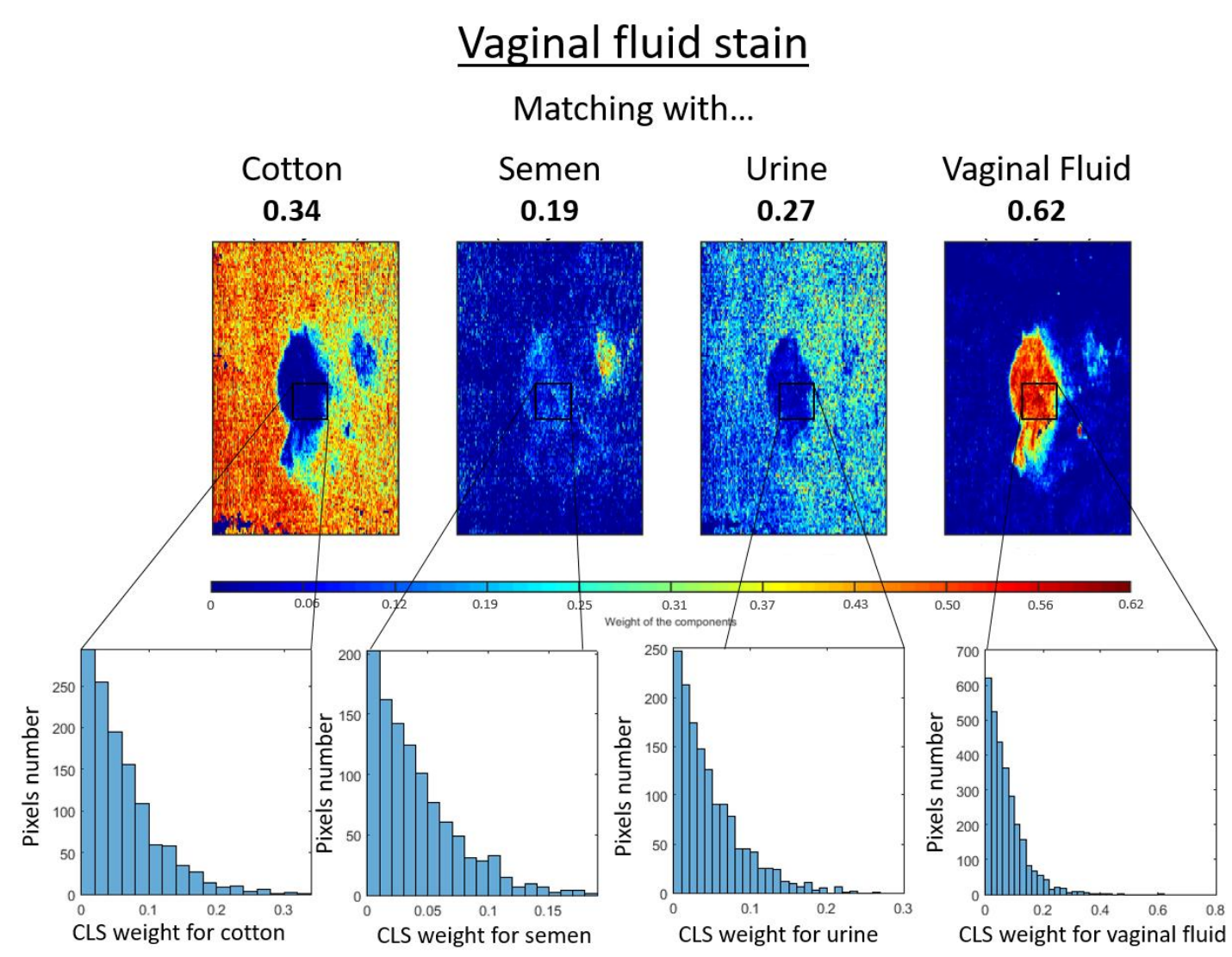

Supplementary Figure S2. CLS classification model applied to a vaginal fluid stain on cotton fabric. The CLS coloured maps for each class (cotton, semen, urine and vaginal fluid) are displayed. The maximum CLS weight values obtained for each class within the selected stained region is indicated above every colour map. The histogram containing the number of pixels within the selected region and their corresponding CLS weight to each class are provided below every colour map.

However, what is the situation when bodily fluids are mixed? Interestingly, both semen and vaginal fluid were independently identified and located within the semen-vaginal fluid mixture-stain. As expected, the coloured map for cotton was highly-red intense outside the stain and highly-blue inside the alleged stained area, which is due to the presence of the bodily fluids (Figure 5). Within this alleged stained region, both semen and vaginal fluid were identified along clearly defined and independent areas, allowing the visualization of the different distributions of semen and vaginal fluid along the stain. Urine, like cotton, was mostly identified outside the stain; but what is more problematic 
is the fact that urine was also identified around some semen regions, which is a negative result since urine was not present in this stain. Finally, in order to check the independent distribution of semen and vaginal fluid two regions were selected for comparison purposes: the first region was selected from an area intensely coloured as semen and vaguely coloured as vaginal fluid whereas the second one was selected from an area intensely marked as vaginal fluid. Positively, the maximum weight and number of pixels for semen and vaginal fluid were in accordance with the colour information of the selected region. For instance, within the first region, a maximum weight value of 0.41 and 2639 pixels were obtained for semen, in comparison with the maximum weight value of 0.22 and 707 pixels obtained for vaginal fluid. On the contrary, within the second region, a maximum weight value of 0.36 and 1576 pixels were obtained for semen, in comparison to the overwhelming maximum weight value of 0.69 and 2122 pixels obtained for vaginal fluid. This way, it becomes extremely clear the optimum spot from which collecting the semen remains containing the aggressor's DNA. Even though both regions contained traces of semen, within the second region these traces were hindered by a considerably larger amount of vaginal fluid. Thus, the first region should be chosen for subsequent genetic analysis, i.e. this region should be the one that might be selected, cut, separated from the rest of the stain with an excess of vaginal fluid, and subsequently subjected to DNA extraction and typing. 


\section{Mixed stain}

Matching with...
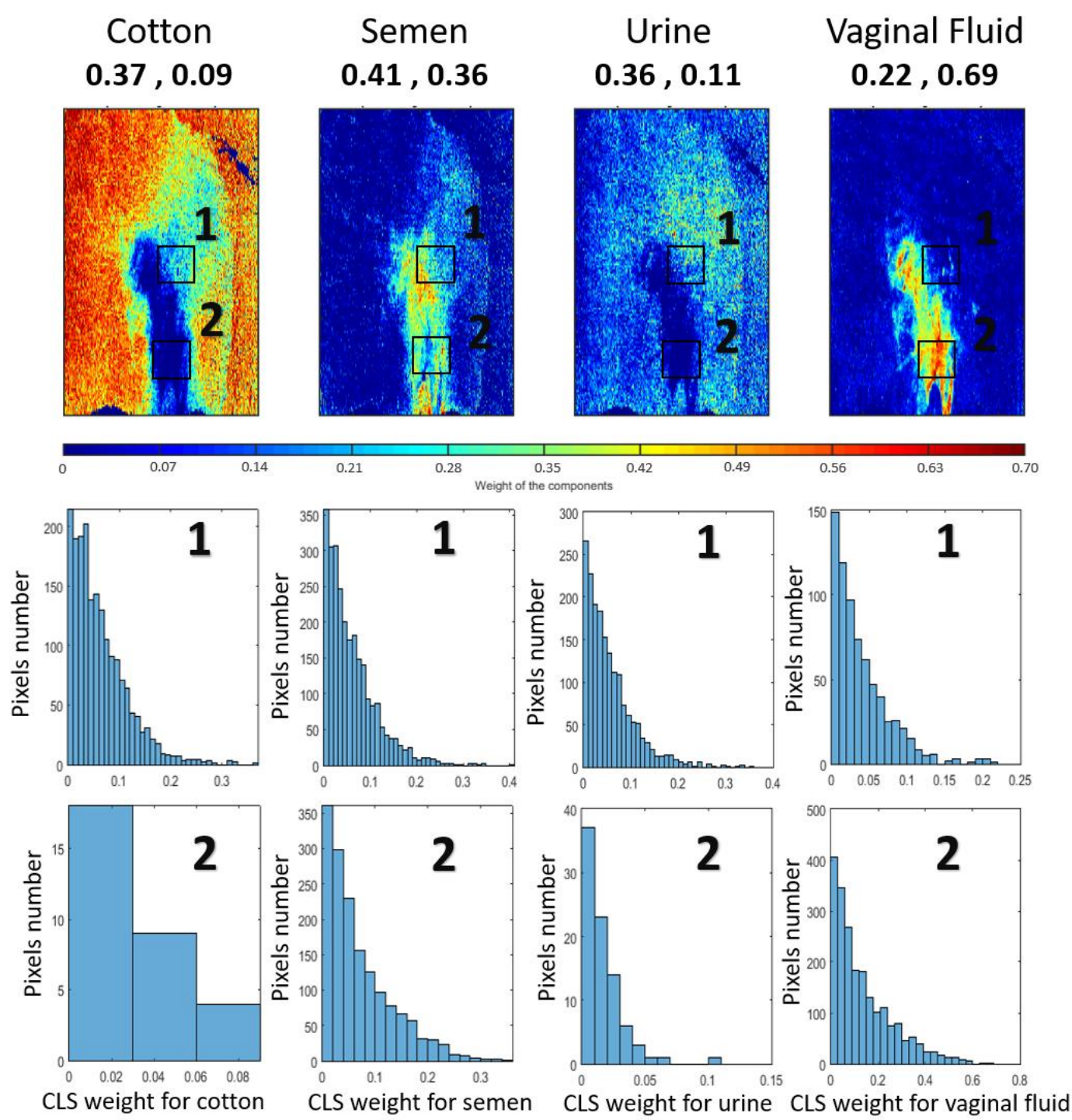

Figure 5. CLS classification model applied to a mixture of semen-vaginal fluid on cotton fabric. The CLS coloured maps for each class (cotton, semen, urine and vaginal fluid) are displayed. The maximum CLS weight values obtained for each class within the two selected stained regions are indicated above every colour map, corresponding the first value to region 1 and the second, to region 2. The histograms containing the number of pixels within the selected regions and their corresponding CLS weight to each class are provided below every colour map.

\section{Conclusions}

First, this study has demonstrated that invisible stains can be easily visualized under NIR wavelengths, especially when using a chemometric analysis such as PCA that easily 
discriminates between stain and cotton (background). In fact, UV-Vis light sources are currently being used for detecting invisible stains, thus it is interesting that NIR can also achieve comparable results.

The most significant capability of NIR-HSI in comparison to UV-Vis is its selectivity, which enabled the discrimination of semen from urine and vaginal fluid; a discrimination whose ultimate goal is the confirmatory identification of different bodily fluids mixed in a unique stain. To this aim, NIR-HSI supported by chemometric classification models such as CLS, has demonstrated to be highly useful to discriminate bodily fluids even in semen-vaginal fluid mixtures which is challenging for forensic practitioners. Thirty years after the discovery and using of UV light sources to non-selectively detect stains of bodily fluids, NIR-HSI has appeared as the perfect complementary technique to overcome the crucial aspect of knowing the distribution of the bodily fluids along the stain. Moreover, this procedure enabled the selective visualization of the individual distribution of each bodily fluid present on the stain providing useful information to select the optimum region from which recovering the semen traces.

According to the results reported in this work, the identification has not been optimum and thus, this methodology (NIR-HSI-CLS) cannot be considered as a confirmatory technique, but it is proposed as useful complementary technique to visualize a stain after having been confirmed traces of semen in it. Further studies using both more advance chemometric tools and larger NIR spectral libraries need to be accomplished to improve the classification and the identification of bodily fluids stains in casework.

\section{Acknowledgements}

We would like to thank Dr. Carolina López and Jordi Alaman (INFAIMON, Barcelona) for kindly providing the hyperspectral camera used in this work. We also thank Dr. $\mathrm{M}^{\mathrm{a}}$ Ángeles Fernández de la Ossa for assisting in the measuring process and Ignacio Jimeno for the assistance with graphic arts. We also acknowledge the project IUICP- 2016-002 from the University Institute of Research in Police Sciences (IUICP). Félix Zapata thanks the Spanish Ministry of Education for his Ph.D. research grant FPU14/00790. 


\section{References}

[1] A. Schepers, Trailer CSI The Hague, 2012, from CSI The Hague project (last accessed September 2016). 〈http://www.csithehague.com/〉.

[2] K. Virkler, I.K. Lednev, Analysis of body fluids for forensic purposes: from laboratory testing to non-destructive rapid confirmatory identification at a crime scene, Forensic Sci. Int. 188 (2009) 1-17.

[3] F. Zapata, M.A. Fernández de la Ossa, C. García-Ruiz, Emerging spectrometric techniques for the forensic analysis of body fluids, Trends Anal. Chem. 64 (2015) $53-63$

[4] F. Zapata, I. Gregório, C. García-Ruiz, Body fluids and spectroscopic techniques in forensics: a perfect match?, J. Forensic Med. 1 (2015).

[5] G.J. Edelman, E. Gaston, T.G. van Leeuwen, P.J. Cullen, M.C.G. Aalders, Hyperspectral imaging for non-contact analysis of forensic traces, Forensic Sci. Int. 223 (2012) 28-39.

[6] D.G. Nelson, K.A. Santucci, An alternate light source to detect semen, Acad. Emerg. Med. 9 (10) (2002) 1045-1048.

[7] N. Vandenberg, R.A.H. van Oorschot, The use of polilight in the detection of seminal fluid, saliva, and bloodstains and comparison with conventional chemical based screening tests, J. Forensic Sci. 51 (2) (2006) 361-370.

[8] A. Fiedler, J. Rehdorf, F. Hilbers, L. Johrdan, C. Stribl, M. Benecke, Detection of semen (human and boar) and saliva on fabrics by a very high powered UV/VIS Light source, Open Forensic Sci. J. 1 (2008) 12-15.

[9] J. Workman Jr., L. Weyer, Practical Guide and Spectral Atlas for Interpretive Near-Infrared Spectroscopy, Second ed., CRC Press, Taylor \& Francis Group, Boca Raton, USA, 2012.

[10] D.A. Burns, E.W. Ciurczak, Handbook of Near Infrared Analysis, Third ed., CRC Press, Taylor \& Francis Group, Boca Raton, USA, 2008.

[11] J.M. Amigo, Practical issues of hyperspectral imaging analysis of solid dosage forms, Anal. Bioanal. Chem. 398 (2010) 93-109.

[12] M. Vidal, J.M. Amigo, Pre-processing of hyperspectral images. Essential steps before image analysis, Chemom. Intell. Lab. Syst. 117 (2012) 138-148.

[13] J.M. Amigo, H. Babamoradi, S. Elcoroaristizabal, Hyperspectral image analysis. A tutorial, Anal. Chim. Acta 896 (2015) 34-51. 
A.C. Lin, H. Hsieh, L. Tsai, A. Linacre, J.C. Lee, Forensic applications of infrared imaging for the detection and recording of latent evidence, J. Forensic Sci. 52 (2007) 1148-1150.

[15] G.J. Edelman, T.G. van Leeuwen, M.C.G. Aalders, Hyperspectral Imaging of the Crime Scene for Detection and Identification of Blood Stains, Proc. SPIE 8743 (2013), 2013 (87430A/1-87430A/7).

[16] B. Li, P. Beveridge, W.T. O'Hare, M. Islam, The application of visible wavelength reflectance hyperspectral imaging for the detection and identification of blood stains, Sci. Justice 54 (2014) 432-438.

[17] G.J. Edelman, T.G. van Leeuwen, M.C. Aalders, Visualization of latent blood stains using visible reflectance hyperspectral imaging and chemometrics, J. Forensic Sci. 60 (2015) S188-S192.

[18] K. Virkler, I.K. Lednev, Raman spectroscopy offers great potential for the nondestructive confirmatory identification of body fluids, Forensic Sci. Int. 181 (1-3) (2008) e1-e5.

[19] V. Sikirzhytski, K. Virkler, I.K. Lednev, Discriminant analysis of Raman spectra for body fluid identification for forensic purposes, Sensors 10 (4) (2010) 2869-2884.

[20] V. Sikirzhytski, A. Sikirzhytskaya, I.K. Lednev, Multidimensional Raman spectroscopic signatures as a tool for forensic identification of body fluid traces: a review, Appl. Spectrosc. 65 (11) (2011) 1223-1232.

[21] K.M. Elkins, Rapid presumptive "Fingerprinting" of body fluids and materials by ATR FT-IR spectroscopy, J. Forensic Sci. 56 (6) (2011) 1580-1587.

[22] C. Orphanou, The detection and discrimination of human body fluids using ATR FT-IR spectroscopy, Forensic Sci. Int. 252 (2015) e10-e16.

[23] F. Zapata, M.A. Fernández, de la Ossa, C. García-Ruiz, Differentiation of body fluid stains on fabrics using external reflection Fourier Transform Infrared Spectroscopy (FT-IR) and chemometrics, Appl. Spectrosc. 70 (4) (2016) 654665.

[24] J.M. Amigo, HYPERTools (〈www.models.life.ku.dk/HYPERTools $\rangle$ ), Department of Food Science, Faculty of Science, University of Copenhagen, Copenhagen, 2016. 